\title{
A Retrospective Analysis of Ruptured Breast Implants
}

\author{
Woo Yeol Baek, Dae Hyun Lew, Dong Won Lee \\ Institute for Human Tissue Restoration, Department of Plastic and Reconstructive Surgery, Yonsei University College of Medicine, Seoul, Korea
}

Background Rupture is an important complication of breast implants. Before cohesive gel silicone implants, rupture rates of both saline and silicone breast implants were over $10 \%$. Through an analysis of ruptured implants, we can determine the various factors related to ruptured implants.

Methods We performed a retrospective review of 72 implants that were removed for implant rupture between 2005 and 2014 at a single institution. The following data were collected: type of implants (saline or silicone), duration of implantation, type of implant shell, degree of capsular contracture, associated symptoms, cause of rupture, diagnostic tools, and management.

Results Forty-five Saline implants and 27 silicone implants were used. Rupture was diagnosed at a mean of 5.6 and 12 years after insertion of saline and silicone implants, respectively. There was no association between shell type and risk of rupture. Spontaneous was the most common reason for the rupture. Rupture management was implant change (39 case), microfat graft (2 case), removal only (14 case), and follow-up loss (17 case).

Conclusions Saline implants have a shorter average duration of rupture, but diagnosis is easier and safer, leading to fewer complications. Previous-generation silicone implants required frequent follow-up observation, and it is recommended that they be changed to a cohesive gel implant before hidden rupture occurs.

Keywords Breast implantation / Silicone gel / Rupture
Correspondence: Dong Won Lee Department of Plastic and Reconstructive Surgery, Yonsei University College of Medicine, 50 Yonsei-ro, Seodaemun-gu, Seoul 120-752, Korea Tel: $+82-2-2228-2215$ Fax: +82-2-393-6947 E-mail:xyphoss@yuhs.ac

No potential conflict of interest relevant to this article was reported.

\section{INTRODUCTION}

Breast implant rupture is one of the most serious complications and concerns of patients. Both manufacturers and physicians commonly explain that breast implants are semi-permanent. However, according to the United States Food and Drug Administration (US FDA) classification, breast implants have a limited product-life. In practice, a breast implant device can maintain its mechanical integrity for decades in a woman's body, but the incidence of rupture increases with time [1]. Upon saline breast im- plant rupturing or leaking, it quickly deflates, which is noticed by the patient. By contrast, rupture of a silicone breast implant does not usually produce a change in volume, and the patient generally cannot realize what has happened in her body [2].

Rupture rates for both saline and silicone breast implants were generally greater than $10 \%$ before the development of fifth-generation silicone gel implants, namely, cohesive gel silicone implants $[3,4]$. The reported mechanisms of breast implant rupture include iatrogenic damage, trauma, chemical degradation of the implant shell, and mechanical pressure during mammography [5]. 
Through the analysis of cases of ruptured implants, one can obtain valuable information about the type of ruptured implant, duration of implantation before rupture, degree of capsular contracture, rupture-associated symptoms, and tools to diagnose ruptured implants. To accomplish this, we examined 72 ruptured breast implants after breast augmentation or reconstruction surgery. We described the relationship between the type of implant and the symptoms associated with a ruptured implant, and evaluated the ruptured period and related assessments. We determined the risk of implant rupture and recommended an approach for follow-up of patients with breast implants.

\section{METHODS}

This study involved a retrospective review of 72 implants, which were removed for suspected implant rupture between 2005 and 2014 at a single institution. The following data were collected for all cases involving both saline and silicone implants: age at rupture diagnosis, duration of implantation before rupture, type of implant shell, degree of capsular contracture, associated symptoms, cause of rupture, tools used to diagnose implant rupture, and management of ruptured implants. Additionally, we analyzed the degree of filling of the saline implants and the type of rupture in the silicone implants.

Shell type was classified as textured, smooth, or unknown (unrecorded). Depending on the deformation of the breast contour, capsular contracture was classified according to the Baker system as grade I, II, III, or IV. Patients' symptoms were classified as size change, skin change, palpable mass, or no symptoms. The cause of rupture was classified as spontaneous, associated with deformity, or iatrogenic. The diagnostic tools used were either ultrasonography or magnetic resonance imaging (MRI). If rupture was confirmed by a surgical procedure without any diagnostic tools, we indicated that the diagnosis was made by symptoms alone. For the method of treatment, we specified whether the patient underwent an implant change, microfat graft, removal only, and follow-up loss.

Many ruptures of breast silicone implants produced no symptoms, and thus, screening tests and MRI findings were used to identify the implant rupture. This study also compared the MRI findings with clinical symptoms and operative findings. During MRI of the breast with a shell rupture, a concave line called the "Linguine" sign was identified, and the rupture was thereby classified as either intra-capsular or extra-capsular.

\section{RESULTS}

The age of the subjects when the implant rupture was diagnosed
Table 1. Patient and breast implant characteristics of 72 ruptured breast implants

\begin{tabular}{|lcc|}
\hline Characteristic & $\begin{array}{c}\text { Saline } \\
(\mathbf{n}=\mathbf{4 5})\end{array}$ & $\begin{array}{c}\text { Silicone } \\
(\mathbf{n}=\mathbf{2 7})\end{array}$ \\
\hline Mean age at diagnosis of ruptured implant (yr) & 48 & 49 \\
Mean duration of implant placement (yr) & 5.6 & 12 \\
Shell type (n) & & \\
$\quad$ Textured & 16 & 11 \\
Smooth & 25 & 4 \\
$\quad$ Unknown & 4 & 12 \\
Capsule Baker stage (n) & & \\
$\quad$ Grade I & 23 & 8 \\
Grade II & 11 & 16 \\
Grade III & 8 & 2 \\
$\quad$ Grade IV & 3 & 1 \\
Purpose of implantation (n) & & \\
$\quad$ Augmentation & 15 & 25 \\
Reconstruction & 30 & 2 \\
\hline
\end{tabular}

ranged from 38 to 52 years. Of the 72 ruptured implants, 45 occurred in saline implants, and 27 occurred in silicone implants. Each ruptured implant was categorized according to the type of implant; the characteristics of the two implant groups are shown in Table 1.

For the saline implants, the mean duration of implantation before diagnosis of the rupture was 5.6 years. The shell type of the ruptured saline implant was textured for 16 cases and smooth for 25 cases. If there were no medical records, the shell type was classified as unknown. Capsulectomy was performed simultaneously in 10 cases, and the following capsular grades were noted: grade I, 23 cases; grade II, 12 cases; grade III, 7 cases; and grade IV, 3 cases. The purpose of implantation was augmentation and reconstruction for 15 and 30 cases, respectively (Table 1). The most common associated symptom in saline implants rupture was size change; other symptoms were much less common. Rupture was primarily diagnosed on the basis of symptoms. Ultrasonography and MRI were much less frequently used for diagnosis. The cause of rupture was predominantly spontaneous in 28 cases, associated with a deformity in 11 cases, and iatrogenic in 6 cases. Capsular contracture of grade III or greater accompanied by deformity was classified as associated with deformity; ruptures occurring during the actual surgery and 6 months after surgery were classified as iatrogenic. The treatment of the rupture was implant change in 35 cases, microfat graft in 2 cases, removal in 2 cases, and nothing in 6 cases. Forty percent of implants were replaced by the same material, whereas $60 \%$ of implants were replaced with silicone (Table 2). The degree of implant filling was most commonly found to be too low (Table 3).

For the silicone implants, the mean duration of implantation until rupture was 12 years. Capsulectomy was executed simulta- 
Table 2. Symptoms, causes, diagnostic tools, and treatment of ruptured implants

\begin{tabular}{|lcc|}
\hline Characteristic & $\begin{array}{c}\text { Saline } \\
(\mathbf{n}=\mathbf{4 5})\end{array}$ & $\begin{array}{c}\text { Silicone } \\
(\mathbf{n}=27)\end{array}$ \\
\hline Symptom & & \\
Size change & $40(80)$ & $6(17)$ \\
Skin change & $3(6)$ & $6(17)$ \\
Palpable mass & $6(12)$ & $14(39)$ \\
No symptom & $1(2)$ & $10(27)$ \\
Cause & & \\
Spontaneous & $28(62)$ & $20(74)$ \\
Associated with deformity & $11(24)$ & $3(11)$ \\
latrogenic & $6(14)$ & $4(15)$ \\
Diagnostic tool & & \\
Symptoms only & $36(80)$ & $0(0)$ \\
Ultrasonography & $5(11)$ & $13(48)$ \\
Magnetic resonance imaging & $4(9)$ & $14(52)$ \\
Treatment & & \\
Implant change & $35(78)$ & $4(15)$ \\
$\quad$ Saline & $14(40)$ & $4(100)$ \\
Silicone & $21(60)$ & $0(0)$ \\
Microfat graft & $2(4)$ & $0(0)$ \\
Removal only & $2(4)$ & $12(44)$ \\
No treatment & $6(14)$ & $11(41)$ \\
\hline Values are presented as number (\%). Multiple assessments were possible be- \\
tween each symptom. & & \\
\hline
\end{tabular}

Table 3. Degree of filling of saline implants

\begin{tabular}{|lc|}
\hline Degree of saline implant filling & Saline \\
\hline Underfilling & 26 \\
Adequate & 7 \\
Overfilling & 4 \\
Unknown & 8 \\
\hline
\end{tabular}

Table 4. Type of rupture of silicone implants

\begin{tabular}{|lc|}
\hline Type of rupture & Silicone \\
\hline Gel bleeda) & 6 \\
Intracapsular & 15 \\
Extracapsular & 6 \\
\hline${ }^{\text {a) Gel bleeding was confirmed during surgery. }}$ \\
\hline
\end{tabular}

neously in 4 cases, and the capsule was graded as follows: grade I, 8 cases; grade II, 15 cases; grade III, 3 cases; and grade IV, 1 case. Two cases involved reconstruction, and 25 involved augmentation surgery. Approximately half of the ruptured silicone implant cases had a history of mammoplasty (Table 1).

For the silicone implants, the most common symptoms of rupture were palpable mass and no symptoms. Fourteen cases (52\%) were diagnosed by MRI, and each reading was found to indicate the opinion as follows (Table 1). Before a rupture was confirmed in silicone implants, a follow-up MRI identified the rupture by implant folding and both intra- and extracapsular

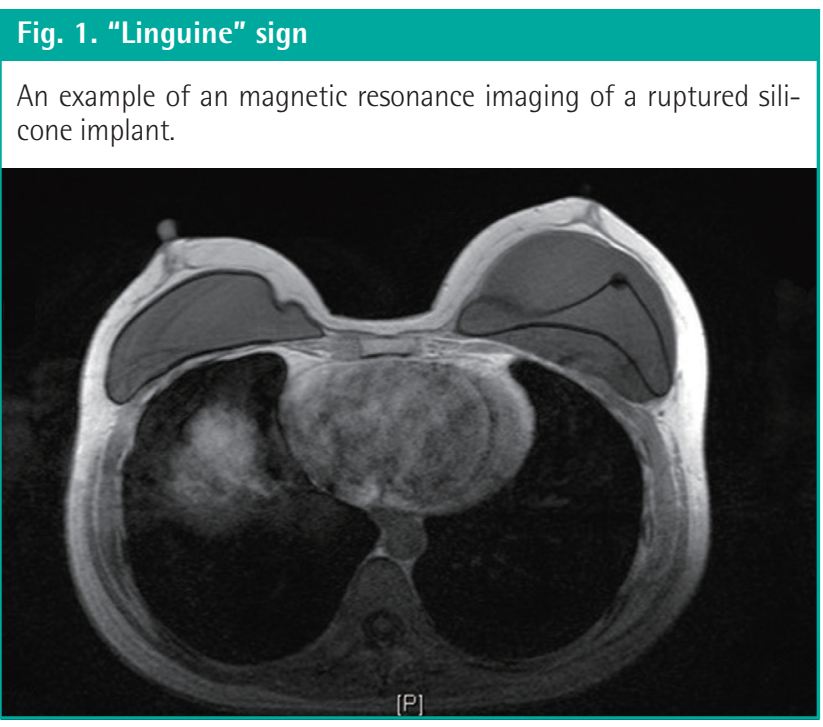

Fig. 2. Ruptured implants

A photograph of the ruptured silicone implant after removal from the body.

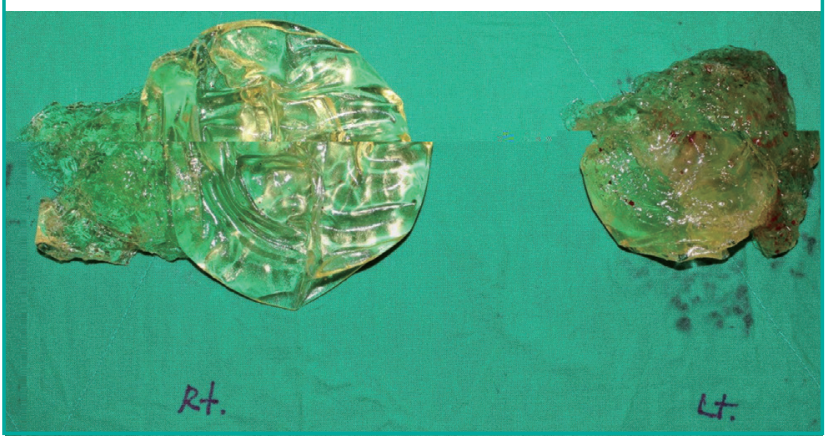

signs of rupture. The most common location of rupture was intra-capsular (15 cases), followed by gel bleed and extra-capsular rupture (6 cases each) (Table 4). Bleeding of the gel, as observed on the image, was not a clear sign of rupture, but if the patient showed symptoms such as tenderness or palpable mass, rupture was diagnosed, which was confirmed after removal of the implant. Fig. 1 shows an example of an MRI image of a ruptured silicone implant. Fig. 2 shows a photograph of the ruptured silicone implant after removal from the body. Treatment after rupture involved implant change in 4 cases, implant removal in 12 cases, and no treatment in 11 cases. All changes to implants were performed using silicone implants (Table 2).

\section{DISCUSSION}

Use of first-generation silicone breast implants was initially reported by the plastic surgeons, Cronin and Gerow [6] in 1962. These silicone breast prostheses, manufactured by Dow Corning Corporation, were filled with moderately viscous silicone 
gel [6]. Fifth-generation silicone gel implants are currently in use, which are made of cohesive silicone gel that typically eliminates filler gel leakage. Saline breast implants, filled with saline solution, were first developed by a French company in 1965 [7]. Although silicone implants require an invasive surgical technique requiring a long surgical incision, the technical goal of using the saline-filled implant was to develop a less-invasive surgical method for inserting an empty implant through a short surgical incision.

Insertion of a breast implant is a common method of breast augmentation and reconstruction surgery, and rupture is a potential complication of all breast implants. Currently available breast implants have different shell thickness. It is difficult to compare types of breast implants according to a standardized measurement, but they can be compared on the basis of number of years used and implant durability [8]. The most substantial difference between saline implants and silicone implants is their ease with which a rupture can be detected, if it occurs. In the numerous studies that have evaluated risk factors for implant rupture, spontaneous rupture has been identified as the main cause of breast implant rupture [9].

Rupture/deflation rates of $3 \%$ to $5 \%$ and $7 \%$ to $10 \%$ have been reported at 3 years and 10 years after insertion of saline breast implants, respectively [3]. Studies on the lifespan of saline implants by Natrelle have shown that rupture occurs in $10 \%$ of these implants during the first 10 years after insertion [3]. This figure can be expected to rise, since the mean time to rupture in our study was approximately 5.6 years. Furthermore, our finding of 48 years old as the mean age when rupture is diagnosed suggests that, considering an average woman's life expectancy of 84.6 years, a saline implant will require replacement in more than $70 \%$ of users during their lifetime [10]. Thus, each institution must closely observe their patients with saline implants. According to the analysis of shell type, saline implant rupture occurred at a higher frequency with a smooth type. However, we did not control other factors like total number of cases, so the results are not statistically significant. Capsular contracture was not used to determine relevance. In a previous study, the shell type of the breast implant was not related to the degree of capsular contracture [11]. Differences in rupture cases between augmentation and reconstruction were attributed to the different numbers of each case. According to a previous study, the implant rupture rate was not statistically significant with regard to the purpose of implantation (Table 1) [12]. Saline implant rupture has been previously reported as being identified and leading to a doctor's visit primarily by the symptom of "sizing down" [13]. In our study, the diagnosis of saline implant rupture was generally accomplished by noting symptoms alone, and the im- plant change rate was high because size change was the most frequent complication. Because a major complication of implantation is a decrease in breast volume, the choice of treatment is to change the implant upon saline implant rupture. Physicians recommend replacement using the same type of previously implanted material or cohesive gel. However, the final selection of the implant type and other treatments was only confirmed after consultation with the patient. For patients who refused the re-insertion of the implant, the implant was removed (Table 2). Furthermore, we observed that ruptured saline implants were associated with under-filling in many instances; however, over-filling and adequate filling were present in approximately $30 \%$ of all cases of implant rupture for which the degree of filling was recorded (Table 3). Authors of a previous study of breast reconstruction suggested that the frequent occurrence of under-filling indicates that under-filling itself is a risk factor for rupture. In that study, although $30 \%$ of all implants were under-filled, under-filling was noted in $70 \%$ of ruptured implants. Thus, in the presence of under-filling, the probability of rupture was increased. Other studies have also suggested that risk factors for saline implant rupture include underfilling, possibly by leading to shell folding [14].

For silicone implants, ruptures were identified at a mean of 12 years after insertion, which was substantially longer than the time for saline implants. This is probably related to the silicone shell being thicker than the saline implant's shell, and the silicone shell's lower likelihood of being damaged during implantation. However, rupture of silicone implants is generally not accompanied by the simultaneous occurrence of symptoms [15]. The rupture itself is usually detected during the process of routine follow-up visits, and when identified in our study, it was primarily at the intra-capsular rupture stage. Gel bleed was not associated with any particular symptoms, and it was thus identified during check-ups [4]. Because gel bleed occurs without noticeable symptoms, it will frequently develop into an intra- or even extracapsular rupture, requiring not only implant removal but often also other procedures. Indeed, additional procedures, such as capsulectomy and foreign body removal, were often performed. A previous study noted that screening tests for silicone implant rupture identified almost 1.9\% [16]. Patients in our study were also suspected during checkups of having a rupture, but because of a lack of relevant symptoms, no procedure was applied in $41 \%$. This can often lead to capsular contracture, siliconoma, and other complications [17]. In cases of ruptured silicone implants, the patients and physician chose implant removal rather than implant re-insertion, because long-term complications like skin color change and a palpable mass were unacceptable to the patient (Table 2). Untreated silicone breast implants have likewise been 
identified in numerous studies as a risk factor for immunologic disorders [18]. The poly implants prostheses (PIP) crisis is an example of problems associated with a particular type of prefilled silicone breast implant: in March 2010, the French government suspended the sale of PIP because of a high failure rate and because of the use of an inappropriate silicone gel [19].

The physical examination is inadequate for correctly evaluating whether a breast implant has ruptured. In asymptomatic patients, only $30 \%$ of ruptured breast implants are accurately detected by palpation by an expert plastic surgeon [20]. In this study, the diagnosis of saline implant rupture was frequently confirmed by changes in the size of the breast. Cases of silicone implant rupture were primarily confirmed using US and MRI. (Table 2) Therefore, many women do not undergo immediate treatment, and more serious complications can consequently arise. The US FDA recommended routine MRI examinations for screening of silent rupture of silicone gel breast implants at 3 years post-implantation and then every 2 years thereafter [21]. MRI is the best diagnostic tool to detect a silicone breast implant rupture. MRI findings are similar to those found during surgery. The MRI signs of rupture have been analyzed extensively studied and important signs, such as the "Linguine" signs, have been identified (Fig. 1). According to long-term MRI data, silicone gel breast implants are associated with silent rupture rates of $9 \%$ to $12 \%$ at 8 years postimplantation [4]. However, the shell of a cohesive gel implant is firmer than that of previous implants, and this filler has a higher viscosity, ranging between that of a liquid and solid. The silicone inside a cohesive gel implant does not flow like water. It will not squirt, or even run freely, even if the tough outer shell is punctured. The cohesive gel tends to remain together. A study of the safety and effectiveness of cohesive gel implants reported low device rupture rates: only $1.1 \%$ at 6 years post-implantation for primary breast augmentation surgery patients [22].

MRI evaluation is expensive, so MRI scans are often obtained only when women have symptoms, or when suggestive findings are noted on other diagnostic tools, such as an ultrasonic study or mammogram [9]. Most detected silicone implant ruptures are found by periodic evaluation for breast cancer. There seem to be many instances of hidden rupture that cause no apparent symptoms. Many ruptured implants exhibited gel bleed and tears, but in those implants retained for over 10 years, many also showed shell thinning (Fig. 2). A breast implant study investigating the anticipated life of silicone implants noted that silicone rupture occurred at a mean patient age of 50 years old. Silicone implants inserted previously should be replaced with fifth-generation cohesive gel before rupture occurs due to shell thinning. If one waits until symptoms of silicone rupture occur, additional procedures and operation time are also increased [17].

Ruptured saline implants have a short life span, but ruptures are easier and safer to diagnose, leading to fewer complications. Rupture of previous-generation silicone implants is often hidden, but it may lead to problems at any time, including an increased risk of cancer. For these older silicone implants, close follow-up is required, in an effort to detect deterioration before rupture occurs. Consideration should be given to changing these older silicone implants to a cohesive gel silicone implant. Cohesive breast implants appear to offer major advantages over their predecessors, but they have been in use for less than 10 years, so further studies are required to evaluate their long-term effects.

\section{REFERENCES}

1. Brown SL, Middleton MS, Berg WA, et al. Prevalence of rupture of silicone gel breast implants revealed on MR imaging in a population of women in Birmingham, Alabama. AJR Am J Roentgenol 2000;175:1057-64.

2. de Camara DL, Sheridan JM, Kammer BA. Rupture and aging of silicone gel breast implants. Plast Reconstr Surg 1993; 91:828-34.

3. Walker PS, Walls B, Murphy DK. Natrelle saline-filled breast implants: a prospective 10-year study. Aesthet Surg J 2009; 29:19-25.

4. Holmich LR, Friis S, Fryzek JP, et al. Incidence of silicone breast implant rupture. Arch Surg 2003;138:801-6.

5. Feng LJ, Amini SB. Analysis of risk factors associated with rupture of silicone gel breast implants. Plast Reconstr Surg 1999;104:955-63.

6. Cronin TD, Gerow FJ. Augmentation mammaplasty: a new "natural feel" prosthesis. In: Transactions of the Third International Congress of Plastic and Reconstructive Surgery; 1963 Oct 13-18; Amsterdam, the Netherlands: Excerpta Medica; 1964. p.41-49.

7. Maxwell GP, Gabriel A. Possible future development of implants and breast augmentation. Clin Plast Surg 2009;36: 167-72.

8. Daniels AU. Silicone breast implant materials. Swiss Med Wkly 2012;142:w13614.

9. Brown SL, Silverman BG, Berg WA. Rupture of silicone-gel breast implants: causes, sequelae, and diagnosis. Lancet 1997;350:1531-7.

10. Yang S, Khang YH, Harper S, et al. Understanding the rapid increase in life expectancy in South Korea. Am J Public Health 2010;100:896-903.

11. Peters W, Keystone E, Smith D. Factors affecting the rupture of silicone-gel breast implants. Ann Plast Surg 1994;32:449- 
51.

12. Levi B, Rademaker AW, Fine NA, et al. Comparison of breast implant deflation for mentor anterior and posterior valve designs in aesthetic and reconstructive patients. Plast Reconstr Surg 2008;122:685-92.

13. Gutowski KA, Mesna GT, Cunningham BL. Saline-filled breast implants: a Plastic Surgery Educational Foundation multicenter outcomes study. Plast Reconstr Surg 1997;100: 1019-27.

14. Lantieri LA, Roudot-Thoraval F, Collins ED, et al. Influence of underfilling on breast implant deflation. Plast Reconstr Surg 1997; 100:1740-4.

15. Rohrich RJ, Reece EM. Breast augmentation today: saline versus silicone-What are the facts? Plast Reconstr Surg 2008;121:669-72.

16. Wang TY, Albornoz C, Hu Q, et al. Abstract 9: a 10-year experience of routine MRI screening for silicone implant rupture: are FDA recommendations supported by evidence? Plast Reconstr Surg 2014;133:978.
17. Holmich LR, Vejborg IM, Conrad C, et al. Untreated silicone breast implant rupture. Plast Reconstr Surg 2004;114: 204-14.

18. Duffy MJ, Woods JE. Health risks of failed silicone gel breast implants: a 30-year clinical experience. Plast Reconstr Surg 1994;94:295-9.

19. Correia-Sa I, Rodrigues-Pereira P, Marques M. The "PIP problem": clinical and histologic characteristics. Aesthetic Plast Surg 2013;37:936-40.

20. Holmich LR, Fryzek JP, Kjoller K, et al. The diagnosis of silicone breast-implant rupture: clinical findings compared with findings at magnetic resonance imaging. Ann Plast Surg 2005;54:583-9.

21. Chung KC, Malay S, Shauver MJ, et al. Economic analysis of screening strategies for rupture of silicone gel breast implants. Plast Reconstr Surg 2012;130:225-37.

22. Cunningham B, McCue J. Safety and effectiveness of Mentor's MemoryGel implants at 6 years. Aesthetic Plast Surg 2009;33:440-4. 